\title{
Attention-Based Capsule Networks with Dynamic Routing for Relation Extraction
}

\author{
Ningyu Zhang ${ }^{1}$ Shumin Deng ${ }^{2,3}$ Zhanlin Sun ${ }^{2} \quad$ Xi Chen ${ }^{2}$ \\ Wei Zhang ${ }^{3,4}$ Huajun Chen ${ }^{2 *}$ \\ 1. Artificial Intelligence Research Institute, Zhejiang Lab, China \\ 2. College of Computer Science and Technology, Zhejiang University, China \\ 3. Alibaba-Zhejiang University Frontier Technology Research Center, China \\ 4. Alibaba Group, China \\ zhangningyu@zju.edu.cn
}

\begin{abstract}
A capsule is a group of neurons, whose activity vector represents the instantiation parameters of a specific type of entity. In this paper, we explore the capsule networks used for relation extraction in a multi-instance multilabel learning framework and propose a novel neural approach based on capsule networks with attention mechanisms. We evaluate our method with different benchmarks, and it is demonstrated that our method improves the precision of the predicted relations. Particularly, we show that capsule networks improve multiple entity pairs relation extraction ${ }^{1}$.
\end{abstract}

\section{Introduction}

This paper focus on the task of relation extraction. One popular method for relation extraction is the multi-instance multi-label learning framework (MIML) (Surdeanu et al., 2012) with distant supervision, where the mentions for an entity pair are aligned with the relations in Freebase (Bollacker et al., 2008). The recently proposed neural network (NN) models (Zeng et al., 2014; Ye et al., 2017; Yang et al., 2018; Wang et al., 2018a) achieve state-of-the-art performance. However, despite the great success of these NNs, some disadvantages remain. First, the existing models focus on, and heavily rely on, the quality of instance representation. Using a vector to represent a sentence is limited because languages are delicate and complex. Second, CNN subsampling fails to retain the precise spatial relationship$\mathrm{s}$ between higher-level parts. The structural relationships such as the positions in sentences are valuable. Besides, existing aggregation operations

\footnotetext{
* Corresponding author.

${ }^{1}$ In this paper, multiple entity pairs relation extraction refers to multiple entity pairs in a single sentence and each pair of entities contains only one relation label.
}

summarizing the sentence meaning into a fixedsize vector such as max or average pooling are lack of guidance by task information. Self-attention (Lin et al., 2017) can select task-dependent information. However, the context vectors are fixed once learned (Gong et al., 2018).

More importantly, most state-of-the-art systems can only predict one most likely relation for a single entity pair. However, it is very common that one sentence may contain multiple entity pairs and describe multiple relations. It is beneficial to consider other relations in the context while predicting the relations (Sorokin and Gurevych, 2017). For example, given the sentence "[Swag It Out] is the official debut single by American [singer] [Zendaya ?", our model can predict multi-relations for these multiple entity pairs simultaneously.

In our work, we present a novel architecture based on capsule networks (Sabour et al., 2017) for relation extraction. We regard the aggregation as a routing problem of how to deliver the messages from source nodes to target nodes. This process enables the capsule networks to decide what and how much information need to be transferred as well as identify complex and interleaved features. Furthermore, the capsule networks convert the multi-label classification problem into a multiple binary classification problem. We also import word-level attention by considering the different contribution of the words. The experimental results show that our model achieves improvements in both single and multiple relation extraction.

\section{Related Work}

Neural Relation Extraction: In the recent years, $\mathrm{NN}$ models have shown superior performance over approaches using hand-crafted features in various tasks. CNN is the first one of the deep learning models that have been applied to relation ex- 


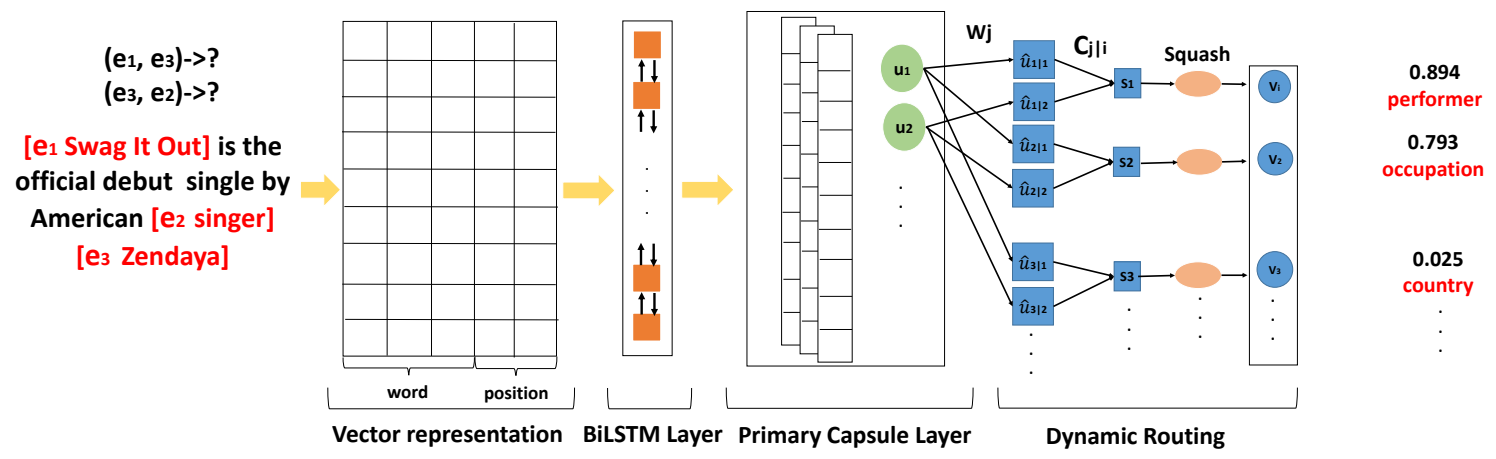

Figure 1: Architecture of capsule networks for relation extraction

traction (Santos et al., 2015). Variants of convolutional networks include piecewise-CNN (PCNN) (Zeng et al., 2015), instance-level selective attention CNN (Lin et al., 2016), rank CNN (Ye et al., 2017), attention and memory CNN (Feng et al., 2017) and syntax-aware CNN (He et al., 2018). Recurrent neural networks (RNN) are another popular choice, and have been used in recent works in the form of attention RNNs (Zhou et al., 2016), context-aware long short-term memory units (LSTMs) (Sorokin and Gurevych, 2017), graph-LSTMs (Peng et al., 2017) and ensemble LSTMs (Yang et al., 2018).

Capsule Network: Recently, the capsule network has been proposed to improve the representation limitations of CNNs and RNNs. (Sabour et al., 2017) replaced the scalar-output feature detectors of CNNs with vector-output capsules and max-pooling with routing-by-agreement. (Hinton et al., 2018)) proposed a new iterative routing procedure among capsule layers, based on the EM algorithm. For natural language processing tasks, (Zhao et al., 2018) explored capsule networks for text classification. (Gong et al., 2018) designed two dynamic routing policies to aggregate the outputs of RNN/CNN encoding layer into a final encoding vector. (Wang et al., 2018b) proposed a capsule model based on RNN for sentiment analysis. To the best of our knowledge, there has been no work that investigates the performance of capsule networks in relation extraction tasks at present.

\section{Methodology}

In this section, we first introduce the MIML framework, and then describe the model architecture we propose for relation extraction, which is shown in Figure 1.

\subsection{Preliminaries}

In MIML, the set of text sentences for the single entity pair or multiple entity pairs ${ }^{2}$ (maximum two entity pairs in this paper) is denoted by $X=$ $\left\{x_{1}, x_{2}, \ldots, x_{n}\right\}$. Assumed that there are $E$ predefined relations (including NA) to extract. Formally, for each relation $r$, the prediction target is denoted by $P\left(r \mid x_{1}, \ldots, x_{n}\right)$.

\subsection{Neural Architectures}

Input Representation: For each sentence $x_{i}$, we use pretrained word embeddings to project each word token onto the $d_{w}$-dimensional space. We adopt the position features as the combination$s$ of the relative distances from the current word to $M$ entities and encode these distances in $M$ $d_{p}$-dimensional vectors ${ }^{3}$. For single entity pair relation extraction, $M=2$; for multiple entity pairs relation extraction, we limit the maximum number of entities in a sentence to four (i.e. two entity pairs). As three entities in one instance is possible when two tuples have a common entity, we set the relative distance to the missing entity to a very large number. Finally, each sentence is transformed into a matrix $x_{i}=$ $\left\{w_{1}, w_{2}, \ldots, w_{L}\right\} \in R^{L \times V}$, where $L$ is the sentence length with padding and $V=d_{w}+d_{p} * M$.

Bi-LSTM Layer: We make use of LSTMs to deeply learn the semantic meaning of a sentence. We concatenate the current memory cell hidden state vector $h_{t}$ of LSTM from two directions as the output vector $h_{t}=\left[\vec{h}_{t}, \overleftarrow{h}_{t}\right] \in R^{2 B}$ at time $t$ where $B$ denotes the dimensionality of LSTM.

\footnotetext{
${ }^{2}$ Since the number of sentences with multiple entity pairs is relatively small, we make use of all the sentences as training samples.

${ }^{3}$ We also adopt an attention mechanism similar to wordlevel attention in Bi-LSTM layer by considering the different contribution of the $M$ position embeddings.
} 
We import word-level attention mechanism as only a few words in a sentence that are relevant to the relation expressed (Jat et al., 2018). The scoring function is $g_{t}=h_{t} \times A \times r$, where $A \in R^{E \times E}$ is a square matrix and $r \in R^{E \times 1}$ is a relation vector. Both $A$ and $r$ are learned. After obtaining $g_{t}$, we feed them to a softmax function to calculate the final importance $\alpha_{t}=\operatorname{softmax}\left(g_{t}\right)$. Then, we get the representation $\tilde{x}_{t}=\alpha_{t} h_{t}$.

For a given bag of sentences, the learning is done using the setting proposed by (Zeng et al., 2015), where the sentence with highest probability of expressing the relation in a bag is selected to train the model in each iteration.

Primary Capsule Layer: Suppose $u_{i} \in R^{d}$ denotes the instantiated parameters set of a capsule, where $d$ is the dimension of the capsule. Let $W^{b} \in R^{2 \times 2 B}$ be the filter shared across different windows. We have a window sliding each 2 -gram vector in the sequence $\tilde{x} \in R^{L \times 2 B}$ with stride 1 to produce a list of capsules $U \in R^{(L+1) \times C \times d}$, totally $C \times d$ filters.

$$
u_{i j}=\operatorname{squash}\left(W_{i}^{b} \otimes S_{j-1: j}+b_{1}\right)
$$

where $0 \leq i \leq C \times d, 0 \leq j \leq L+1$, $\operatorname{squash}(x)=\frac{\|x\|^{2}}{0.5+\|x\|^{2}} \frac{x}{\|x\|}, b_{1}$ is the bias ter$\mathrm{m}$. For all $C \times d$ filters, the generated capsule feature maps can be rearranged as $U=$ $\left\{u_{1}, u_{2}, \ldots, u_{(L+1) \times C}\right\}$, where totally $(L+1) \times C$ $d$-dimensional vectors are collected as capsules.

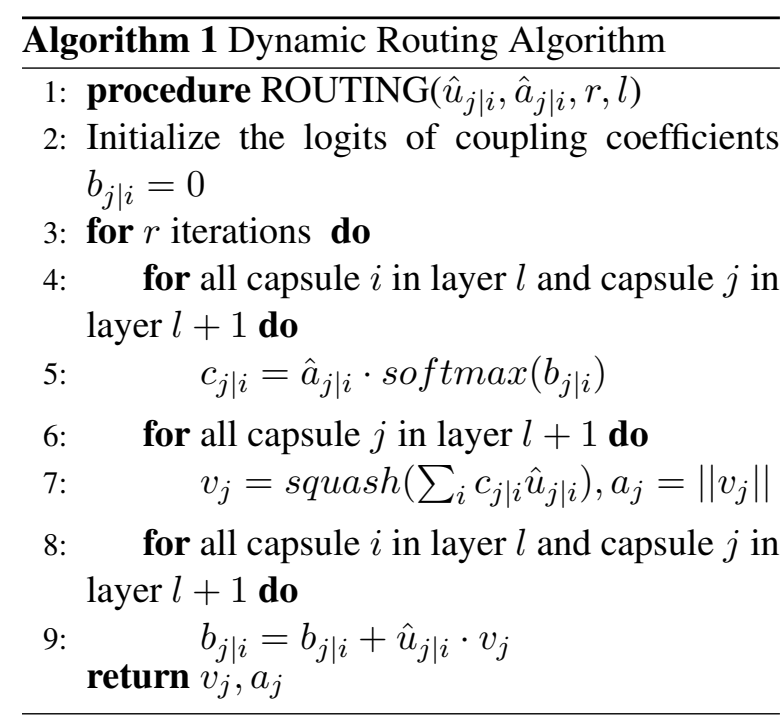

Dynamic Routing: We explore the transformation matrices to generate the prediction vector $u_{j \mid i} \in R^{d}$ from a child capsule $i$ to its parent capsule $j$. The transformation matrices share weights $W^{c} \in R^{E \times d \times d}$ across the child capsules, where
$E$ is the number relations (parent capsules) in the layer above. Formally, each corresponding vote can be computed by:

$$
\hat{u}_{j \mid i}=W_{j}^{c} u_{i}+\hat{b}_{j \mid i} \in R^{d}
$$

The basic idea of dynamic routing is to design a nonlinear map:

$\left\{\hat{u}_{j \mid i} \in R^{d}\right\}_{i=1, \ldots, H, j=1, \ldots, E} \mapsto\left\{v_{j} \in R^{d}\right\}_{j=1}^{E}$

where $H=(L+1) \times C$.

Inspired by (Zhao et al., 2018), we attempt to use the probability of existence of parent capsules to iteratively amend the connection strength, which is summarized in Algorithm 1. The length of the vector $v_{j}$ represents the probability of each relation. We use a separate margin loss $L_{k}$ for each relation capsule $k$ :

$$
\begin{aligned}
L_{k} & =Y_{k} \max \left(0, m^{+}-\left\|v_{k}\right\|\right)^{2} \\
& +\lambda\left(1-Y_{k}\right) \max \left(0,\left\|v_{k}\right\|-m^{-}\right)^{2}
\end{aligned}
$$

where $Y_{k}=1$ if the relation $k$ is present, $m^{+}=$ $0.9, m^{-}=0.1$ and $\lambda=0.5$. The total loss can be formulated as: $L_{\text {total }}=\sum_{k=1}^{E} L_{k}$

\subsection{Prediction}

For single entity pair relation extraction, we calculate the length of the vector $v_{j}$ which represents the probability of each relation. For multiple entity pairs relation extraction, we choose relations with top two probability meanwhile bigger than the threshold (We empirically set the threshold 0.7). Finally, we may get one or two predicted relations $r$. Given entity pair $\left(e_{1}, e_{2}\right)$, in order to choose which relationship the tuple belongs to, we adopt the pretrained embeddings of entities and relations ${ }^{4}$ and calculate $r_{k}=\underset{k}{\arg \min }\left|t-h-r_{k}\right|$ , where $t, h$ are the embeddings of entities $e_{1}, e_{2}$ respectively and $r_{k}$ is the relation embedding. The relation with the closest embedding to the entity embedding difference is the predicted category.

\section{Experiments}

We test our model on the NYT dataset (NYT) developed by (Riedel et al., 2010) for single entity pair relation extraction and the Wikidata dataset (Sorokin and Gurevych, 2017) for multiple entity pairs relation extraction. We exclude sentences longer than $L$. All code is implemented in Tensorflow (Abadi et al., 2016). We adopt the Adam

\footnotetext{
${ }^{4}$ http://openke.thunlp.org
} 
optimizer (Kingma and $\mathrm{Ba}$, 2014) with learning rate 0.001 , batch size 128 , LSTMs' unit size 300 , $L=120, d_{p}=5, d=8, C=32$, dropout rate 0.5 , routing iteration 3 .

\subsection{Practical Performance}

NYT dataset (Single entity pair):

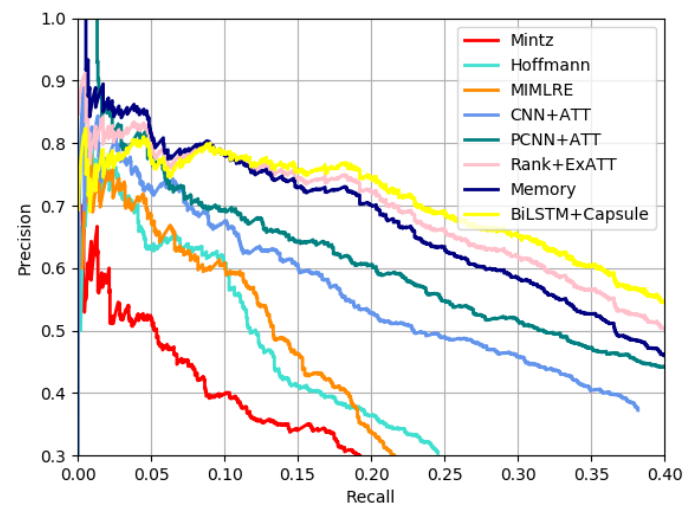

Figure 2: Performance comparison on the NYT dataset.

We utilize the word embeddings released by (Lin et al., 2016) ${ }^{5}$. The precision-recall curves for different models on the test set are shown in Figure 2. Our model BiLSTM+Capsule achieves comparable results compared with all baselines, where Mintz refers to (Mintz et al., 2009), Hoffmann refers to (Hoffmann et al., 2011), MIMLRE refers to (Surdeanu et al., 2012), CNN+ATT refers to (Zeng et al., 2015), PCNN+ATT refers to (Lin et al., 2016), Rank+ExATT refers to (Ye et al., 2017) and Memory refers to (Feng et al., 2017). We also show the precision numbers for some particular recalls as well as the AUC in Table 1, where our model generally leads to better precision. Interestingly, we observe our model achieve comparable results to predict multi-relation compared with Rank+ExATT in Figure 3. Given an entity tuple (South Korea, Seoul) which has two relations: /location/./administrative_divisions and /location/./capital. We observe these two relations have the highest scores among the other relation$s$ in our model which demonstrate the ability of multi-relations prediction.

Wikidata dataset (Multiple entity pairs):

We train word embeddings using Glove (Pennington et al., 2014) ${ }^{6}$ on the Wikipedia Corpus.

$$
\begin{aligned}
{ }^{5} d_{w} & =50 \\
{ }^{6} d_{w} & =200
\end{aligned}
$$

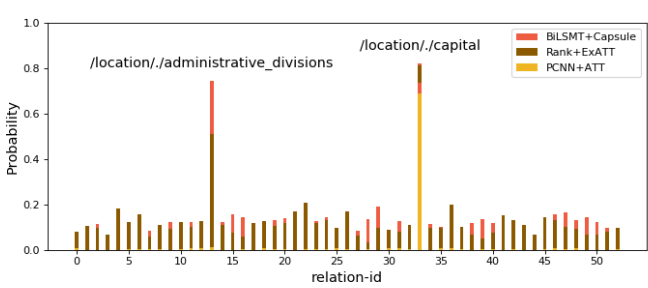

Figure 3: Normalized output relation scores.

Table 1: Precisions on the NYT dataset.

\begin{tabular}{c|c|c|c|c|c}
\hline \hline Recall & 0.1 & 0.2 & 0.3 & 0.4 & AUC \\
\hline PCNN+ATT & 0.698 & 0.606 & 0.518 & 0.446 & 0.323 \\
\hline Rank+ExATT & 0.789 & 0.726 & 0.620 & 0.514 & 0.395 \\
\hline Our Model & 0.788 & $\mathbf{0 . 7 4 3}$ & $\mathbf{0 . 6 5 4}$ & $\mathbf{0 . 5 4 6}$ & $\mathbf{0 . 3 9 7}$ \\
\hline \hline
\end{tabular}

We show the precision numbers for some particular recalls as well as the AUC in Table 2, where PCNN+ATT (1) refers to train sentences with two entities and one relation label, PCNN+ATT (m) refers to train sentences with four entities ${ }^{7}$ and two relation labels. We observe that our model exhibits the best performances. Moreover, in the process of predicting the existence of relations for a sentence, our approach is more convenient, as the PCNNATT (1) has to predict all possible pairs of entities in the sentence while our approach can predict multiple relations simultaneously.

Table 2: Precisions on the Wikidata dataset.

\begin{tabular}{c|c|c|c|c}
\hline \hline Recall & 0.1 & 0.2 & 0.3 & AUC \\
\hline Rank+ExATT & 0.584 & 0.535 & 0.487 & 0.392 \\
\hline PCNN+ATT (m) & 0.365 & 0.317 & 0.213 & 0.204 \\
\hline PCNN+ATT (1) & 0.665 & 0.517 & 0.413 & 0.396 \\
\hline Our Model & 0.650 & 0.519 & 0.422 & $\mathbf{0 . 4 0 5}$ \\
\hline \hline
\end{tabular}

Ablation study: To better demonstrate the performance of capsule net and attention mechanism, we remove the primary capsule layer and dynamic routing to make Bi-LSTM layer followed by a fully connected layer instead. We also remove the word-level attention separately. The experimental results on Wikidata dataset are summarized in Table 3. The results of "-Word-ATT" row refers to the results without word-level attention. According to the table, the drop of precision demonstrates that the word-level attention is quite useful. Generally, all two proposed strategies contribute to the effectiveness of our model.

\footnotetext{
${ }^{7}$ Two additional position embeddings.
} 
Table 3: Ablation study of capsule net and word-level attention on Wikidata dataset.

\begin{tabular}{c|c|c|c|c}
\hline Recall & 0.1 & 0.2 & 0.3 & AUC \\
\hline -Word-ATT & 0.648 & 0.515 & 0.395 & 0.389 \\
\hline -Capsule & 0.635 & 0.507 & 0.413 & 0.386 \\
\hline Our Model & 0.650 & 0.519 & 0.422 & 0.405 \\
\hline
\end{tabular}

\subsection{Discussion}

CNN vs Capsule: Capsule networks achieve comparable results compared with baselines. In fact, the capsule combines features by clustering. A nonlinear map is constructed in an iterative manner, ensuring the output of each capsule to be sent to an appropriate parent in the subsequent layer. Dynamic routing may be more effective than the strategies such as max-pooling in C$\mathrm{NN}$, which essentially detects whether a feature is present in any position of the text or not, but loses spatial information of the feature. Additionally, capsule achieves comparable results to predict multi-relations in the case of single entity pair, and performs better in the case of multiple entity pairs relation extraction.

Choice of $d$ : In the experiments, the larger the dimension of the capsule, the more the capabilities of the feature information it contains. However, larger dimension increases the computational complexity. We test different levels of dimensions of capsules. The model is trained on two Nvidia GTX1080ti GPUs with 64G RAM and six Intel(R) Core(TM) i7-6850K CPU 3.60GHz. As the table 4 depicts, the training time increases with the growth of $d$. When $d=32$, we observe that the loss decreases very slowly and the model is difficult to converge. So we only train 2 epochs and stop training. We set the parameter $d=8 \mathrm{em}$ pirically to balance the precision and training time cost.

Table 4: Precisions on the Wikidata dataset with different choice of $d$.

\begin{tabular}{c|c|c|c|c|l}
\hline Recall & 0.1 & 0.2 & 0.3 & AUC & Time \\
\hline$d=1$ & 0.602 & 0.487 & 0.403 & 0.367 & $4 \mathrm{~h}$ \\
\hline$d=32$ & 0.645 & 0.501 & 0.393 & 0.370 & - \\
\hline$d=16$ & 0.655 & 0.518 & 0.413 & 0.413 & $20 \mathrm{~h}$ \\
\hline$d=8$ & 0.650 & 0.519 & 0.422 & 0.405 & $8 \mathrm{~h}$ \\
\hline
\end{tabular}

\section{Effects of Iterative Routing:}

We also study how the iteration number affec$t$ the performance on the Wikidata dataset. Table
Table 5: Precisions on the Wikidata dataset with different number of dynamic routing iterations.

\begin{tabular}{c|c|c|c|c}
\hline Recall & 0.1 & 0.2 & 0.3 & $\mathrm{AUC}$ \\
\hline Iteration=1 & 0.531 & 0.455 & 0.353 & 0.201 \\
\hline Iteration=2 & 0.592 & 0.498 & 0.385 & 0.375 \\
\hline Iteration=3 & 0.650 & 0.519 & 0.422 & 0.405 \\
\hline Iteration=4 & 0.601 & 0.505 & 0.422 & 0.385 \\
\hline Iteration=5 & 0.575 & 0.495 & 0.394 & 0.376 \\
\hline
\end{tabular}

5 shows the comparison of 1 - 5 iterations. We find that the performance reach the best when iteration is set to 3 . The results indicate the dynamic routing is contributing to improve the performance. Specifically, in the iteration algorith$\mathrm{m}$, the $b_{j \mid i}=b_{j \mid i}+\hat{u}_{j \mid i} \cdot v_{j}$. When the number of iteration is very large, $v_{j}$ becomes either 0 or 1 , which means each underlying capsule is only linked to a single upper capsule. Therefore, the iteration times should not be too large.

\subsection{Conclusion}

We propose a relation extraction approach based on capsule networks with attention mechanism. Although we use Bi-LSTM as sentence encoding in this paper, the other encoding method, such as convolved $\mathrm{n}$-gram, could be alternatively used. Experimental results of two benchmarks show that the model improves the precision of the predicted relations.

In the future, we tend to resolve the situation of how to assign predicted relationship to multi entity pairs when two entities have multi-relations by utilizing prior knowledge such as entity type and joint training with named entity recognition. We will also try to optimize the model in terms of speed and focus on other problems by leveraging class ties between labels, specially on multilabel learning problems. Besides, dynamic routing could also be useful to improve other natural language processing tasks such as the sequenceto-sequence task and so on.

\section{Acknowledgments}

We want to express gratitude to the anonymous reviewers for their hard work and kind comments, which will further improve our work in the future. This work is funded by NSFC 61673338/61473260, and partly supported by Alibaba-Zhejiang University Joint Institute of Frontier Technologies. 


\section{References}

Martín Abadi, Paul Barham, Jianmin Chen, Zhifeng Chen, Andy Davis, Jeffrey Dean, Matthieu Devin, Sanjay Ghemawat, Geoffrey Irving, Michael Isard, et al. 2016. Tensorflow: A system for large-scale machine learning. In $O S D I$, volume 16, pages 265 283.

Kurt Bollacker, Colin Evans, Praveen Paritosh, Tim Sturge, and Jamie Taylor. 2008. Freebase: a collaboratively created graph database for structuring human knowledge. In Proceedings of the 2008 ACM SIGMOD international conference on Management of data, pages 1247-1250. AcM.

Xiaocheng Feng, Jiang Guo, Bing Qin, Ting Liu, and Yongjie Liu. 2017. Effective deep memory networks for distant supervised relation extraction. In Proceedings of the Twenty-Sixth International Joint Conference on Artificial Intelligence, IJCAI, pages 19-25.

Jingjing Gong, Xipeng Qiu, Shaojing Wang, and Xuanjing Huang. 2018. Information aggregation via dynamic routing for sequence encoding. arXiv preprint arXiv:1806.01501.

Zhengqiu He, Wenliang Chen, Zhenghua Li, Meishan Zhang, Wei Zhang, and Min Zhang. 2018. See: Syntax-aware entity embedding for neural relation extraction. arXiv preprint arXiv:1801.03603.

Geoffrey Hinton, Nicholas Frosst, and Sara Sabour. 2018. Matrix capsules with em routing.

Raphael Hoffmann, Congle Zhang, Xiao Ling, Luke Zettlemoyer, and Daniel S Weld. 2011. Knowledgebased weak supervision for information extraction of overlapping relations. In Proceedings of the 49th Annual Meeting of the Association for Computational Linguistics: Human Language TechnologiesVolume 1, pages 541-550. Association for Computational Linguistics.

Sharmistha Jat, Siddhesh Khandelwal, and Partha Talukdar. 2018. Improving distantly supervised relation extraction using word and entity based attention. arXiv preprint arXiv: 1804.06987.

Diederik P Kingma and Jimmy Ba. 2014. Adam: A method for stochastic optimization. arXiv preprint arXiv:1412.6980.

Yankai Lin, Shiqi Shen, Zhiyuan Liu, Huanbo Luan, and Maosong Sun. 2016. Neural relation extraction with selective attention over instances. In Proceedings of the 54th Annual Meeting of the Association for Computational Linguistics (Volume 1: Long Papers), volume 1, pages 2124-2133.

Zhouhan Lin, Minwei Feng, Cicero Nogueira dos Santos, Mo Yu, Bing Xiang, Bowen Zhou, and Yoshua Bengio. 2017. A structured self-attentive sentence embedding. arXiv preprint arXiv:1703.03130.
Mike Mintz, Steven Bills, Rion Snow, and Dan Jurafsky. 2009. Distant supervision for relation extraction without labeled data. In Proceedings of the Joint Conference of the 47th Annual Meeting of the $A C L$ and the 4th International Joint Conference on Natural Language Processing of the AFNLP: Volume 2-Volume 2, pages 1003-1011. Association for Computational Linguistics.

Nanyun Peng, Hoifung Poon, Chris Quirk, Kristina Toutanova, and Wen-tau Yih. 2017. Cross-sentence n-ary relation extraction with graph lstms. arXiv preprint arXiv:1708.03743.

Jeffrey Pennington, Richard Socher, and Christopher Manning. 2014. Glove: Global vectors for word representation. In Proceedings of the 2014 conference on empirical methods in natural language processing (EMNLP), pages 1532-1543.

Sebastian Riedel, Limin Yao, and Andrew McCallum. 2010. Modeling relations and their mentions without labeled text. In Joint European Conference on Machine Learning and Knowledge Discovery in Databases, pages 148-163. Springer.

Sara Sabour, Nicholas Frosst, and Geoffrey E Hinton. 2017. Dynamic routing between capsules. In $A d$ vances in Neural Information Processing Systems, pages 3859-3869.

Cicero Nogueira dos Santos, Bing Xiang, and Bowen Zhou. 2015. Classifying relations by ranking with convolutional neural networks. arXiv preprint $\operatorname{arX}$ iv: 1504.06580 .

Daniil Sorokin and Iryna Gurevych. 2017. Contextaware representations for knowledge base relation extraction. In Proceedings of the 2017 Conference on Empirical Methods in Natural Language Processing, pages 1784-1789.

Mihai Surdeanu, Julie Tibshirani, Ramesh Nallapati, and Christopher D Manning. 2012. Multi-instance multi-label learning for relation extraction. In Proceedings of the 2012 joint conference on empirical methods in natural language processing and computational natural language learning, pages 455-465. Association for Computational Linguistics.

Guanying Wang, Ruoxu Wang, and Huajun Chen. 2018a. Label-free distant supervision for relation extraction via knowledge graph embedding. In Proceedings of the 2018 Conference on Empirical Methods in Natural Language Processing.

Yequan Wang, Aixin Sun, Jialong Han, Ying Liu, and Xiaoyan Zhu. 2018b. Sentiment analysis by capsules. In Proceedings of the 2018 World Wide Web Conference on World Wide Web, pages 1165-1174. International World Wide Web Conferences Steering Committee.

Dongdong Yang, Senzhang Wang, and Zhoujun Li. 2018. Ensemble neural relation extraction with adaptive boosting. arXiv preprint arXiv:1801.09334. 
Hai Ye, Wenhan Chao, Zhunchen Luo, and Zhoujun Li. 2017. Jointly extracting relations with class ties via effective deep ranking. In Proceedings of the 55th Annual Meeting of the Association for Computational Linguistics (Volume 1: Long Papers), volume 1, pages 1810-1820.

Daojian Zeng, Kang Liu, Yubo Chen, and Jun Zhao. 2015. Distant supervision for relation extraction via piecewise convolutional neural networks. In Proceedings of the 2015 Conference on Empirical Methods in Natural Language Processing, pages 17531762.

Daojian Zeng, Kang Liu, Siwei Lai, Guangyou Zhou, and Jun Zhao. 2014. Relation classification via convolutional deep neural network. In Proceedings of COLING 2014, the 25th International Conference on Computational Linguistics: Technical Papers, pages 2335-2344.

Wei Zhao, Jianbo Ye, Min Yang, Zeyang Lei, Suofei Zhang, and Zhou Zhao. 2018. Investigating capsule networks with dynamic routing for text classification. arXiv preprint arXiv:1804.00538.

Peng Zhou, Wei Shi, Jun Tian, Zhenyu Qi, Bingchen $\mathrm{Li}$, Hongwei Hao, and Bo Xu. 2016. Attentionbased bidirectional long short-term memory networks for relation classification. In Proceedings of the 54th Annual Meeting of the Association for Computational Linguistics (Volume 2: Short Papers), volume 2, pages 207-212. 